\title{
Reduction of persistent air leak with endoscopic valve implants
}

\section{Tudor P Toma, Onn Min Kon, William Oldfield, Reina Sanefuji, Mark Griffiths, Frank Wells, Siva Sivasothy, Michael Dusmet, Duncan M Geddes, Michael I Polkey}

The standard management of air leaks due to persistent bronchopleural fistula involves chest drainage and occasionally pleurodesis, with intractable cases requiring surgical decortication or surgical repair. However, some of these patients may be at high risk for surgery, particularly if they have already had thoracic surgery or have other medical problems; for this group there is a need for less invasive methods of stopping or reducing air leaks. Emphasys endobronchial valves (EBV) are occlusive devices designed primarily for endoscopic lung volume reduction in emphysema. Because the device is a one-way inspiratory airway blocker, it is possible that it could be used in controlling persistent air leaks while maintaining the drainage of secretions. Two cases are reported of persistent air leaks that were managed by endoscopic occlusion with EBV. In one case complete stoppage of the air leak was achieved with immediate clinical benefits. The second patient died 5 days after treatment from additional complications apparently not related to the procedure. Endobronchial blockage may be a useful salvage procedure for patients with persistent air leak for whom there is no other treatment available.

A ir leaks are frequent after pulmonary resection, but can occur as a complication in a diseased lung or even in a previously normal lung. A number of methods are available to treat these, and specific algorithms have been recommended..$^{1-3}$ The standard management of persistent air leaks involves chest drainage and occasionally pleurodesis, with intractable cases requiring surgical decortication or surgical repair. ${ }^{4}$ However, some of these patients may be at high risk for such an intervention, particularly if they have already had thoracic surgery or have other medical problems; for this group there is a need for less invasive methods of stopping or reducing air leaks.

When temporary bronchoscopic occlusion of a segmental or subsegmental bronchus can be shown to interrupt the air leak, permanent occlusion of this airway can be performed. A number of bronchial blocking devices have been used for this purpose, and include the use of glues, stents and coils. ${ }^{4-11}$ Watanabe Spigots (Novatech, Cedex, France) are the only bronchial blocker specifically made to reduce a persistent air leak by occluding the affected bronchus, but their use requires specific technical experience and is limited to Japan. ${ }^{12}$

Emphasys (Emphasys Medical Inc, California, USA) endobronchial valves (EBV) are occlusive devices designed primarily for endoscopic lung volume reduction in emphysema. ${ }^{13}$ They function as a selective block to the inspiratory air flow and have a valve that allows drainage in expiration. The initial safety and efficacy of Emphasys valves in emphysema is encouraging. ${ }^{14}$ Because the device is a one-way inspiratory airway blocker, we hypothesised that it could also be used for controlling persistent air leaks while maintaining the drainage of secretions. The clinical data on two patients are presented here. The technique was also used successfully in a third patient who is not presented here because we were unable to obtain consent to publicly disclose the medical details.

\section{METHODS \\ Determination of affected bronchi}

Although the bronchi that conduct the air to the leak may be broadly surmised from a previous surgical examination or from a CT scan, this type of clinical opinion is less likely to identify bronchial subdivisions leading to a leak. To achieve maximal efficacy in one of our two patients, the affected bronchi were therefore detected more precisely by the use of a test occlusion of the suspected bronchial segment using a balloon catheter, as previously described by Watanabe et al. ${ }^{12}$ Briefly, a balloon catheter (B5-2C, Olympus KeyMed, Southend on Sea, UK) was passed through the working channel of the flexible bronchoscope and inflated to achieve complete occlusion of the tested segment. If the air coming through the chest drain was diminished or stopped by keeping the occlusion for $1-3$ min, then the source of leakage was attributed to the particular segment tested.

\section{Valve insertion}

Bronchial occlusion was performed with the Emphasys EBV and delivery catheter according to protocols described elsewhere for patients with emphysema. ${ }^{13}$ The classic valve (CEBV) wase used in one patient and the transcopic valve (TSEBV) in the second (figs 1 and 2). The two types of valve are similar in function but the TS-EBV can be delivered through a catheter that is small enough to go through the working channel of a flexible bronchoscope. All procedures were performed under general anaesthesia.

The bronchoscopy was performed through the endotracheal tube in one patient. The second patient had a tracheostomy tube and the bronchoscope was inserted through the mouth and passed alongside the endotracheal portion of the tracheostomy tube and its cuffing balloon; the volume of air in the cuff was decreased but the balloon was not completely deflated for the passage of the bronchoscope.

Following bronchial occlusion, the valves were left in situ and no attempts were made to remove them.

\section{CASE REPORTS}

Case 1

A 35-year-old woman with lymphangioleiomyomatosis was treated with a single lung transplant. Immediately after surgery her level of respiratory support was increased and she required a modified Monaldi decompressing procedure on her native lung. ${ }^{15}$ This resulted in a high flow air leak originating from the anterior segment of the left upper lobe, treated with a chest drain placed under water seal. Because the flow of her air leak was not diminishing, a request was made by the transplant

Abbreviations: $C-E B V$, classic endobronchial valve; $E B V$, endobronchial valve; EWS, endoscopic Watanabe spigot; NSAID, non-steroidal antiinflammatory drug; TS-EBV, transcopic endobronchial valve 


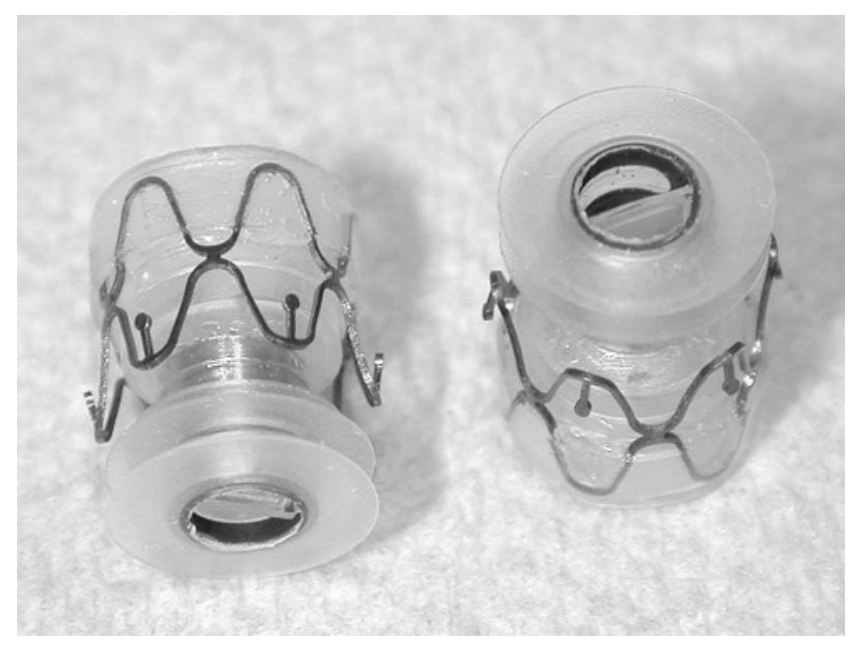

Figure 1 Classic endoscopic valve implant inserted with the help of a guidewire.

team to attempt occlusion of the anterior bronchial segment of her native left upper lobe.

The source of the air leak was known and bronchial occlusion was started with valve placement in the anterior segment of the left upper lobe, without being preceded by a balloon test. After valve insertion there was no immediate reduction of the air flow at the chest drain. After multidisciplinary evaluation, it was decided to attempt a second procedure with the aim of achieving complete occlusion of the left upper lobe and lingula. The second bronchoscopy was done $48 \mathrm{~h}$ after the first procedure, and three more valves were inserted to completely occlude the left upper lobe and lingula. Immediately after the procedure the air leak stopped with no bubbling at the chest drain. However, within the next $4 \mathrm{~h}$, air drainage re-occurred but at much lower flows. The patient remained stable in the short term but died 5 days after the second procedure. The cause of death (confirmed by a post mortem examination) was cardiorespiratory arrest due to mucus plugging and a new pneumothorax on the transplanted lung.

\section{Case 2}

A 32-year-old woman developed a bilateral pneumothorax following severe community acquired pneumonia and adult

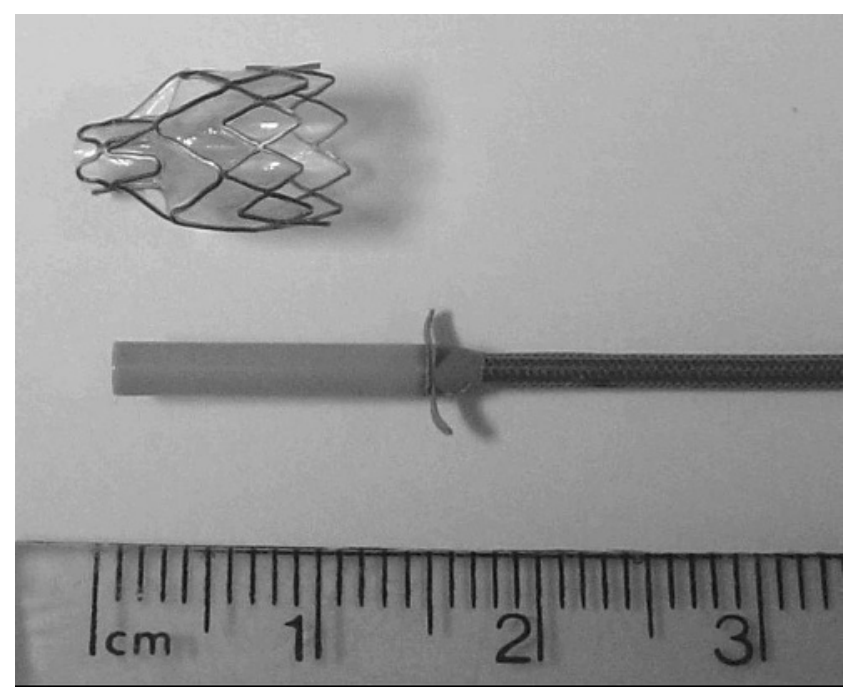

Figure 2 Transcopic valve implant inserted directly through the working channel of a flexible bronchoscope. respiratory distress syndrome. In addition, she had a complicated past medical history of postnatal depression and eating disorders which had led to a Bilroth-1 gastrectomy for a nonhealing pyloric ulcer secondary to use of non-steroidal antiinflammatory drugs (NSAID), NSAID enteropathy with protein losing state, profound hypoalbuminaemia, oedema and hypoglobulinaemia.

She was initially treated conservatively with a chest drain under water seal; the air leak on the left stopped, but a high rate of air leak on the right persisted. One month after her pneumothorax there was no improvement in her condition and, following a respiratory consultation, occlusion of her right upper lobe was attempted using the balloon catheter passed through a bronchoscope. However, this failed to reduce the air leak and no valve insertion was attempted on that occasion.

Her management proceeded with a right muscle-sparing thoracotomy and decortication, with removal of the sixth rib and debridement of a large right upper lobe abscess. The air leak was visualised and sutured using prolene. Two intercostal drains were inserted at the time of the procedure. She remained stable postoperatively and initially the air leak stopped completely, but after a few days it again became substantial. At the same time as the leak increased there was a degree of collapse of the right upper lobe with fully expanded middle and lower lobes.

There was slow but steady progress with the level of ventilatory support required, and the patient was gradually weaned from BIPAP to spontaneous ventilation using a tracheostomy mask over the course of 6 weeks. During this period she had an episode of sepsis secondary to Pseudomonas aeruginosa which was successfully treated with antibiotics.

Although she had clinically improved, there was no radiological improvement of the right pneumothorax and therefore insertion of the balloon was reattempted. This time the balloon was successful in stopping the air leak completely. The balloon was left in situ as a temporary method of occlusion, but shortly after the procedure the patient had episodes of coughing which resulted in displacement of the balloon. However, as it was proved that the bronchopulmonary fistula had reduced in size sufficiently to be mechanically blocked, she was offered placement of Emphasys valves in the right upper lobe. Three TS-EBVs were placed to completely occlude all three segments of her right upper lobe. The procedure was successful in stopping the air leak within $2 \mathrm{~h}$ of completion of the occlusion. Her ventilation after the procedure improved and within 1 week she was decannulated, transferred to a general ward and made a good recovery, which continued during the next weeks. Once the air leak had resolved her drains were successfully removed.

At clinic review 12 months after the procedure she had a residual airspace visible on the chest radiographs but was otherwise well and living at home. Chest radiographs taken before and after treatment are shown in figs 3 and 4 . She was most recently reviewed 22 months after the procedure because of infection in the residual airspace which resolved with antibiotics.

\section{DISCUSSION}

Here we describe two patients who underwent endoscopic occlusion with Emphasys valves for the treatment of persistent air leaks refractory to conventional treatments and their immediate follow-up. In one case (case 2), complete stoppage of the air leak was achieved with immediate clinical benefits. In the other case the air leaks were considerably reduced, but the patient died from additional complications apparently not related to the procedure. Endobronchial blockage with valve 


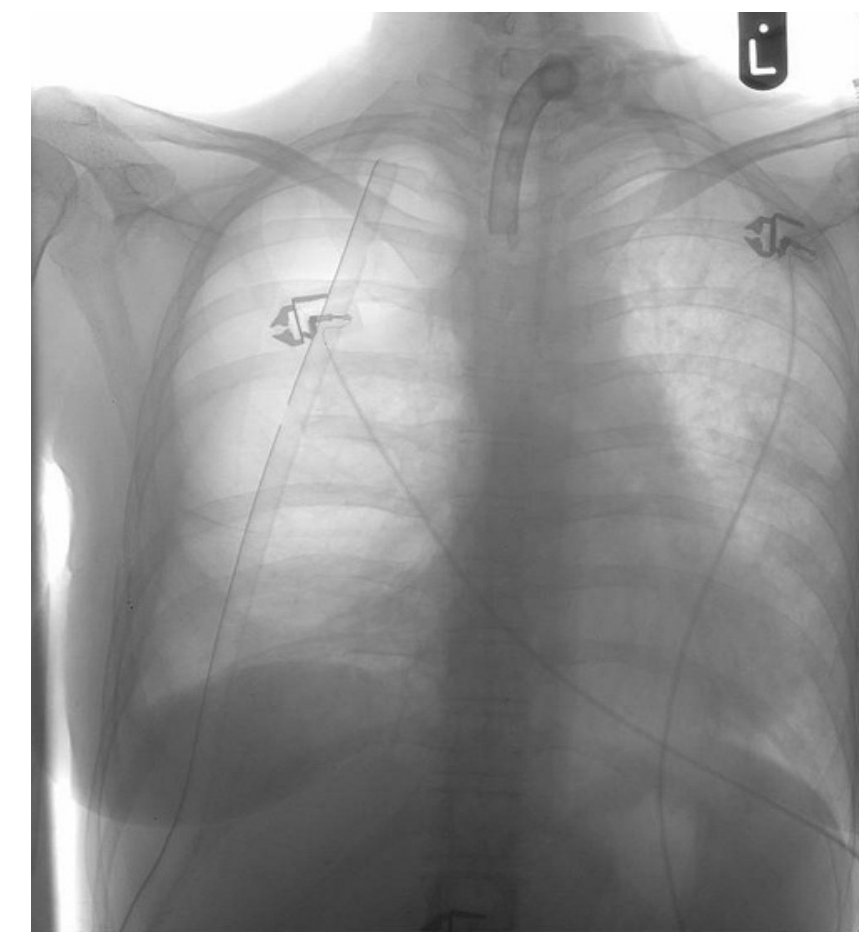

Figure 3 Chest radiograph of patient 2 before valve insertion.

implants appears to be a possible salvage procedure for patients with persistent air leak.

The choice of C-EBV or TS-EBV was primarily based on the equipment available at the time of the procedures. However, both types of valves could be placed in these severely compromised patients without technical difficulties and no procedural complications. Both types of devices were well tolerated and did not migrate; moreover, in the surviving case there were no additional infective complications. Because of their ease of insertion and removal, the TS-EBV should probably be the device of choice if available. No attempt was made to remove the devices because in case 2 the lung did not re-expand completely.

The clinical progression of these patients was influenced by the condition that led to the occurrence of the leak. The death of patient 1 did not appear to be related to the valve insertion at post mortem examination, and the type of fatal complications observed were not unexpected for patients undergoing a transplant; moreover, these complications occurred after an interval of stability after valve insertion. In case 2 the clinical benefit was apparent immediately and the improvement was rapid.

Watanabe et al considered using silicone bronchial fillers (endoscopic Watanabe spigots, EWS) on 63 patients including 40 cases of intractable pneumothorax, 12 of pyothorax with bronchial fistula, 7 of pulmonary fistula, 3 of bronchial fistula with other organs, and 1 case of bronchial fistula. Bronchial occlusion with EWS was tried in 60 cases and technical success was obtained in $58(96.7 \%)$. Air leakage was stopped in 23 cases $(39.7 \%)$ and reduced in $22(37.9 \%)$. Reported complications included 2 cases $(3.3 \%)$ of dyspnoea, 2 (3.3\%) of pneumonia and 1 case $(1.7 \%)$ of fever. However, the 2 cases of pneumonia were not related to this procedure and were cured by the administration of antibiotics. ${ }^{12}$ Compared with EWS, the Emphasys valves have a different mechanism of insertion and a unidirectional valve that allows drainage of air and secretions from the isolated lobe. Although Emphasys valves appear to be easier to insert and have a valve mechanism against secretion accumulation, there are no data to suggest that blocking the airways with valves is safer or more effective than with EWS.

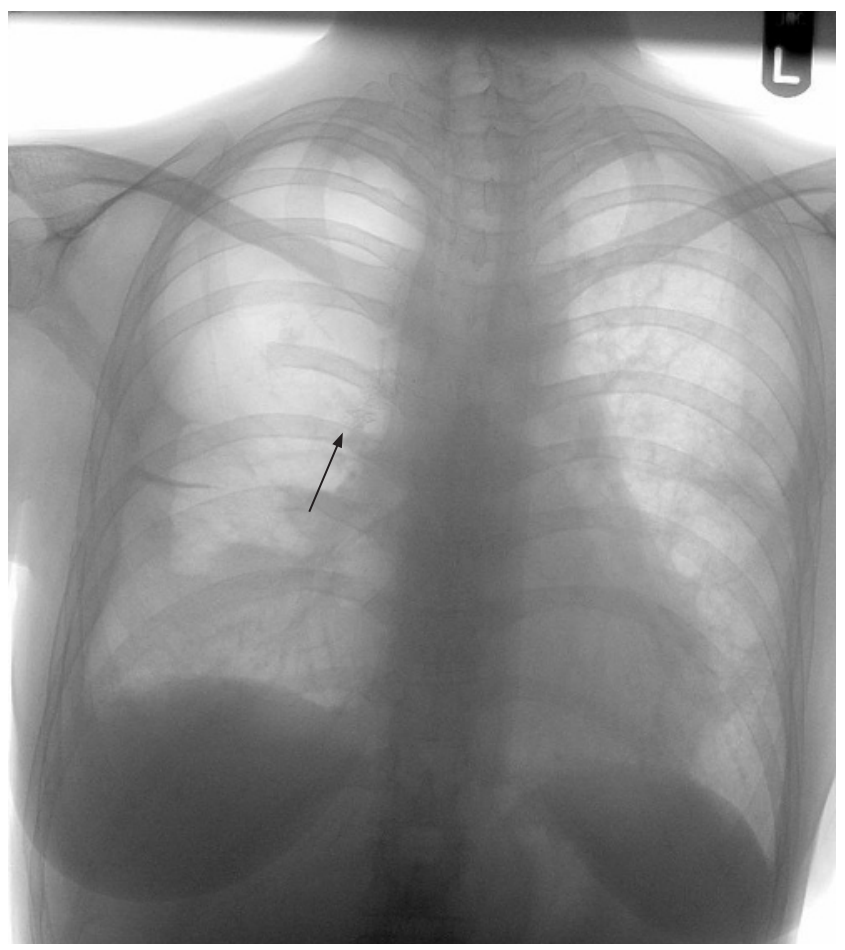

Figure 4 Chest radiograph of patient 2 seven days after valve insertion. Three valves (arrow) are visible in the right upper lobe; the chest drain and the tracheal tube were removed.

A bronchopleural fistula is a communication between a mainstem, lobar or segmental bronchus and the pleural space and implies pulmonary resection, while an alveolar-pleural fistula is a communication between the pulmonary parenchyma distal to a segmental bronchus and the pleural space. ${ }^{2}$ The patients treated here both had air leaks due to alveolar-pleural fistulas; the role of the valves in the treatment of postoperative bronchopleural fistulas is unknown. Reports exist of the use of glues, stents and coils in the treatment of bronchopleural fistulas. ${ }^{4-11}$ In theory, the main advantage of the valve system over other forms of simple bronchial occlusion in the treatment of air leaks may be the presence of the valve which allows drainage and its ease of removal if it becomes necessary.

The role of EBVs in the treatment of persistent air leaks will depend on their cost which, at present, is unknown. The average cost per day in a UK intensive care unit (ICU) is estimated to be $£ 964$ ( $€ 1406)$, with a range of $£ 883-1241$ $(€ 1288-1809) .{ }^{16}$ The final commercial price of the valves will probably be determined by the outcome of the ongoing multicentre study evaluating their use in emphysema. Nevertheless, only a modest reduction in ICU stay would be necessary for them to be cost effective.

The observations described here suggest that valves can be an additional tool in the armamentarium of treatments for persistent air leaks, where other methods of treatment have failed or in frail patients at high risk of surgery. Randomised prospective studies in larger groups are needed to determine the safety and efficacy and cost effectiveness of the valves in this group of patients.

\section{Authors' affiliations}

T P Toma, R Sanefuji, M Griffiths, M Dusmet, D M Geddes, M I Polkey, Royal Brompton Hospital, London, UK

O M Kon, W Oldfield, Chest and Allergy Clinic, St Mary's Hospital, London, UK

F Wells, S Sivasothy, Papworth Hospital, Cambridge, UK 
Emphasys Medical provided the valves free of charge and an unrestricted educational grant to Royal Brompton Hospital, but they had no part in the analysis of data or in the preparation of this manuscript.

Correspondence to: Dr Tudor P Toma, Department of Respiratory Medicine, Lewisham University Hospital, Lewisham High Street, London SE136LA, UK; Homa@doctors.org.uk

Received 30 March 2005

Accepted 15 March 2006

\section{REFERENCES}

1 Cerfolio RJ, Tummala RP, Holman WL, et al. A prospective algorithm for the management of air leaks after pulmonary resection. Ann Thorac Surg 1998;66:1726-31

2 Cerfolio RJ, Bass CS, Pask AH, et al. Predictors and treatment of persistent air leaks. Ann Thorac Surg 2002;73:1727-30.

3 Cerfolio RJ, Bass C, Katholi CR. Prospective randomized trial compares suction versus water seal for air leaks. Ann Thorac Surg 2001;71:1613-7.

4 Puskas JD, Mathisen DJ, Grillo HC, et al. Treatment strategies for bronchopleural fistula. J Thorac Cardiovasc Surg 1995;109:989-96.

5 Lin J, lannettoni MD. Closure of bronchopleural fistulas using albuminglutaraldehyde tissue adhesive. Ann Thorac Surg 2004;77:326-8

6 Takanami I. Closure of a bronchopleural fistula using a fibrin-glue coated collagen patch. Interactive Cardiovasc Thorac Surg 2003;2:387-8.
7 Baumann WR, Ulmer JL, Ambrose PG, et al. Closure of a bronchopleural fistula using decalcified human spongiosa and a fibrin sealant. Ann Thorac Surg 1997:64:230-3.

8 Menard JW, Prejean CA, Tucker WY. Endoscopic closure of bronchopleural fistulas using a tissue adhesive. Am J Surg 1988;155:415-6.

9 Glover W, Chavis TV, Daniel TM. Fibrin glue application through the flexible fiberoptic bronchoscope: closure of bronchopleural fistulas. J Thorac Cardiovasc Surg 1987;93:470-2.

10 Watanabe S-I, Watanabe T, Urayama H. Endobronchial occlusion method of bronchopleural fistula with metallic coils and glue. Thorac Cardiovasc Surgeon 2003;51:106-8.

11 Uchida T, Wada M, Sakamoto J, et al. Treatment for empyema with bronchopleural fistulas using endobronchial occlusion coils: report of a case. Surgery Today 1999;29:186-9.

12 Watanabe Y, Matsuo K, Tamaoki A, et al. Bronchial occlusion with endobronchial Watanabe spigot. J Bronchol 2003;10:264-7.

13 Toma TP, Polkey MI, Goldstraw PG, et al. Methodological aspects of bronchoscopic lung volume reduction with a proprietary system. Respiration 2003:70:658-64.

14 Toma TP, Hopkinson NS, Hillier J, et al. Bronchoscopic volume reduction with valve implants in patients with severe emphysema. Lancet 2003;361:931-3.

15 Venn GE, Williams PR, Goldstraw P. Intracavity drainage for bullous, emphysematous lung disease: experience with the Brompton technique. Thorax 1988;43:998-1002.

16 Jacobs P, Rapoport J, Edbrooke D. Economies of scale in British intensive care units and combined intensive care/high dependency units. Intensive Care Med 2004;30:660-4.

\section{LUNG ALERT}

\section{Strategies for reducing treatment in mild persistent asthma}

$\Delta$ American Lung Association Asthma Clinical Research Centers, Peters SP, Anthonisen N, et al. Randomised comparison of strategies for reducing treatment in mild persistent asthma. N Engl J Med 2007;356:2027-39.

nhaled corticosteroids (ICS) are standard treatment for persistent asthma but it is important to "step down" treatment when possible to minimise adverse effects. The optimal strategy for reducing treatment in mild asthma has not yet been determined.

Five hundred patients with mild asthma (pre-bronchodilator $\mathrm{FEV}_{1}>60 \%$ predicted) well controlled on 4-6 weeks fluticasone $100 \mu \mathrm{g}$ twice daily were included in this randomised, double-blind controlled trial. Subjects were randomised to either continued treatment with fluticasone at the same dose, montelukast $5-10 \mathrm{mg}$ once daily or fluticasone $100 \mu \mathrm{g}$ once daily and salmeterol (SALM) $50 \mu \mathrm{g}$ once daily for 16 weeks. The primary outcome measure was time to treatment failure (hospitalisation, systemic corticosteroid use, lung function decline or increased use of rescue treatment). Secondary outcome measures included asthma symptom scores.

Treatment failure was significantly higher in the montelukast group compared with the other two groups $(\mathrm{p}=0.03)$. The commonest reason in all groups for failure was a fall in $\mathrm{FEV}_{1} \geqslant 20 \%$ of the baseline value. Patients treated with montelukast had more frequent nocturnal awakenings than those on fluticasone and worse Asthma Control Questionnaire scores than both groups. However, the montelukast group had fewer upper respiratory infections than both groups and less upper gastrointestinal disturbance than the fluticasone group. The percentage of symptomfree days and other secondary outcome measures were similar in all three groups.

This study provides a rationale for step down in treatment in mild asthma to low dose combined fluticasone-SALM, potentially lowering the mean cumulative ICS dose and adverse effects of ICS. The authors have not discussed the potential effects of the addition of salmeterol and the study does not tell us whether a step down to once daily fluticasone without salmeterol would be effective. 